\title{
Inverse Square Law in Spectrally Bounded Quantum Dynamics
}

\author{
Er'el Granot \\ Department of Electrical and Electronics Engineering, Ariel University, Ariel, Israel \\ Email: erel@ariel.ac.il
}

How to cite this paper: Granot, E. (2019) Inverse Square Law in Spectrally Bounded Quantum Dynamics. Journal of Applied Mathematics and Physics, 7, 2701-2711. https://doi.org/10.4236/jamp.2019.711184

Received: October 1, 2019

Accepted: November 5, 2019

Published: November 8, 2019

Copyright $\odot 2019$ by author(s) and Scientific Research Publishing Inc. This work is licensed under the Creative Commons Attribution International License (CC BY 4.0).

http://creativecommons.org/licenses/by/4.0/ (c) (i) Open Access

\begin{abstract}
The object of the paper is to formulate Quantum (Schrödinger) dynamics of spectrally bounded wavefunction. The Nyquist theorem is used to replace the wavefunction with a discrete series of numbers. Consequently, in this case, Schrödinger dynamics can be formalized as a universal set of ordinary differential Equations, with universal coupling between them, which are related to Euler's formula. It is shown that the coefficient $(m, n)$ is inversely proportional to the distance between the points $n$ and $m$. As far as we know, this is the first time that this inverse square law was formulated.
\end{abstract}

\section{Keywords}

Quantum Dynamics, Schrödinger Equation, Inverse Square Law, Euler Formula, Quantum Causality

\section{Introduction}

Unlike in Maxwell's wave Equation, where perturbations propagate at the speed of light, in Schrödinger dynamics, any local perturbation is instantaneously felt all over space, just as in the diffusion Equation [1]. However, unlike the diffusion Equation where the nonlocal effect is exponentially small, in the Schrödinger Equation, it decays much slower-as a power law.

In both cases, i.e., in the diffusion and the Schrödinger cases, the causality is violated due to the asymmetry between space and time.

In the Klein-Gordon's (KG), or similarly in the Dirac's, Equation, due to the symmetry between space and time, causality reappears. In the KG case, the nonlinear dispersion relation distorts the wavefunction in high agreement with the Schrödinger Equation only as far as causality allows, i.e., as far as the distance $x=c t$ from the local perturbation [2] [3]. That is, any local perturbation af- 
fects over the entire $x= \pm c t$ domain. Clearly, in the non-relativistic regime (i.e., the Schrödinger case) this domain is the entire space. As a result, an initial discontinuous wavefunction can kindle currents all over space instantaneously [4] [5].

There are several numerical methods to calculate non-relativistic quantum mechanics dynamics. Every method emphasizes a different feature of this dynamics. The Schrödinger Equation is a differential Equation and therefore it emphasizes the locality nature of the dynamics (see, for example, Ref. [6]). Consequently, it is not optimized to evaluate the dynamics of singular, discontinuous or even sharp functions. Eigenstates expansion, in general, and Fourier expansion, in particular, is a powerful technique when the eigenstates, as well as their boundary conditions, are well-known (see, for example, Ref. [7]). It is inconvenient to use this method when the boundary conditions are ill-defined, such as in scattering problems. Path integrals method is an elegant method to formulate the wavefunction as a superposition of infinite paths [8]. Due to its complexity, it is mainly used for pedagogical purposes to explain the nature of particle interferences (or with conjunction with Monte-Carlo simulation [6]). When other options are possible, such as kernel methods, they are usually preferred. Kernel, or Green function, methods are very useful in cases where the initial wavefunction is given [8] [9]. However, if the wavefunction is spread all over space, the kernel's oscillations frequencies, which increase indefinitely may cause numerical difficulties.

In this paper we discuss the case, where the initial wavefunction is spectrally bounded, i.e., the results of any energy measurement are bounded by a certain value. In this case, all the information of the wavefunction can be encapsulated in a series of discrete values. Consequently, the quantum dynamics can be written as an exact infinite set of ordinary differential Equations. It will be shown that the couplings between these Equations consist of universal numbers, which inversely depend on the spatial distance between points on the wavefunction.

\section{The Dynamics}

The differential version of the free Schrödinger Equation

$$
i \hbar \frac{\partial \psi(x, t)}{\partial t}=-\frac{\hbar^{2}}{2 m} \frac{\partial^{2} \psi(x, t)}{\partial x^{2}}
$$

sometimes conceals the nonlocal properties of the Schrödinger dynamics. However, its integral presentation

$$
\psi(x, t)=\int_{-\infty}^{\infty} K\left(x-x^{\prime}, t\right) \psi\left(x^{\prime}, 0\right) \mathrm{d} x^{\prime}
$$

with the free-space Schrödinger Kernel [8]

$$
K\left(x-x^{\prime}, t\right)=\sqrt{\frac{m}{2 \pi i \hbar t}} \exp \left[\frac{i m}{2 \hbar} \frac{\left(x-x^{\prime}\right)^{2}}{t}\right]
$$


illustrates the nonlocality more vividly.

Nevertheless, the oscillations' frequency increase so rapidly that their averages (which is equivalent to the integral operation) quickly converges to zero and the locality properties reappear. In particular, in some cases, the latter nonlocal Equation (2) was used to derive the former local one (1) [8].

A local analysis of the Schrödinger Equation is an excellent approximation in the quasi-classical regimes, which mathematically equivalent to the stationary phase approximation [10]. However, the locality is questionable in the quantum regime. The problem is that Equation (3) is the impulse response of the Schrödinger Equation (1), i.e., it is the quantum system's response to the initial state of a delta function. However, a delta function can never be a physical state (it is based on infinite energies and it is not normalizable). To take a more physical initial state, it is usually accustomed to replacing the impulse response with a more physical, finite-width pulse-response, i.e.,

$$
\psi(x, 0)=\frac{1}{\rho^{1 / 2}}\left(\frac{2}{\pi}\right)^{1 / 4} \exp \left(-x^{2} / \rho^{2}\right)
$$

In which case the pulse response (after a period $t$ )

$$
\psi(x, t)=\frac{1}{\sqrt{1-i 2 \hbar t \rho^{-2} / m} \rho^{1 / 2}}\left(\frac{2}{\pi}\right)^{1 / 4} \exp \left(-\frac{x^{2}}{\left(1-i 2 \hbar t \rho^{-2} / m\right) \rho^{2}}\right)
$$

decays (in space) exponentially as well.

Therefore, it may seem as if the locality approximation is justified since in principle one can choose $\rho$ to be arbitrarily small. However, this result is based on the premises that the spatial spectrum of the wavefunction is unbounded.

When the spatial spectrum of the wavefunction is bounded, i.e., when the spectral coefficient beyond the spatial frequency domain $|k|<\pi / \Delta x$ are all zero ( $k$ represents the wavenumber), then, according to the Nyquist theorem, the wavefunction can be written as a superposition of sinc functions [11]. That is, all the information in the wavefunction can be written as an infinite discrete series of complex numbers $\psi_{n}=\mathfrak{R} \psi_{n}+i \Im \psi_{n}$ for $n=-\infty, \cdots,-1,0,1,2, \cdots, \infty$. In the spectral domain, the wavefunction occupies the spatial spectral bandwidth $1 / \Delta x$. Consequently, from the Nyquist theorem, the initial wavefunction can be written as an infinite sequence of overlapping Nyquist-sinc functions (for applications in the optical communication sphere see Ref. [12]-[17]) (see Figure 1), i.e.,

$$
\psi(x, t=0)=\sum_{n=-\infty}^{\infty} \psi_{n} \operatorname{sinc}(x / \Delta x-n),
$$

where $\operatorname{sinc}(\xi) \equiv \frac{\sin (\pi \xi)}{\pi \xi}$ is the "sinc" function, which corresponds to the Fourier transform of the rectangular function (the spectral shape of the channel). 

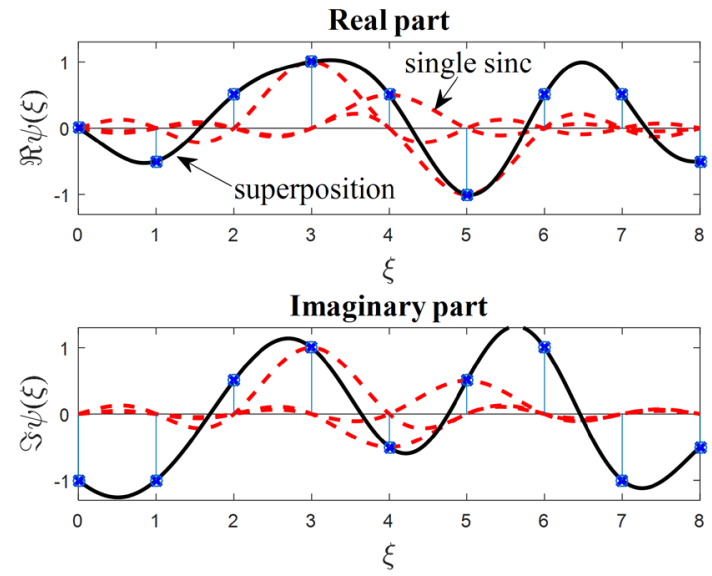

Figure 1. Illustration of the method, in which any spectrally bounded function can be written as an infinite series of sinc pulses. In the figure the sinc pulses, which are centered at $\xi=x / \Delta x=3,4$ and 5 are plotted by dashed curves, while the final function is presented by solid curves (real/imaginary part in the upper/lower panel). The dashed curves represent single sinc's, while the solid curve represents the superposition function. The Upper panel represents the real part of the wavefunction, while the lower one represents its imaginary component.

Due to the linear nature of the system, Equation (6) can be solved directly

$$
\psi(x, t>0)=\sum_{n=-\infty}^{\infty} \psi_{n} \mathrm{~d} \operatorname{sinc}\left(x / \Delta x-n,(\hbar / m) t / \Delta x^{2}\right)
$$

where "dsinc" is the dynamic-sync function [18]

$$
\operatorname{dsinc}(\xi, \tau) \equiv \frac{1}{2} \sqrt{\frac{i}{2 \pi \tau}} \exp \left(-i \frac{\xi^{2}}{2 \tau}\right)\left[\operatorname{erf}\left(-\frac{\xi-\pi \tau}{\sqrt{i 2 \tau}}\right)-\operatorname{erf}\left(-\frac{\xi+\pi \tau}{\sqrt{i 2 \tau}}\right)\right] \text {. }
$$

Clearly, $\lim _{\tau \rightarrow 0}[\operatorname{dsinc}(\xi, \tau)]=\operatorname{sinc}(\xi)$.

To simplify the derivation we use the dimensionless variables

$$
\tau \equiv(\hbar / m) t / \Delta x^{2} \text { and } \xi \equiv x / \Delta x .
$$

Some of the properties of the dsinc function are illustrated in Figure 2 and Figure 3. As can be seen, the distortions from the initial delta function dsinc $(n, 0)=\delta(n)$ gradually increase with time $(\tau)$.

With notations (9) at hand, Equations (1) and (7) can be rewritten

$$
i \frac{\partial \psi(\xi, \tau)}{\partial \tau}=-\frac{1}{2} \frac{\partial^{2} \psi(\xi, \tau)}{\partial \xi^{2}}
$$

and

$$
\psi(\xi, \tau>0)=\sum_{n=-\infty}^{\infty} \psi_{n} \mathrm{~d} \operatorname{sinc}(\xi-n, \tau)
$$

respectively.

\section{Matrix Formulation}

When $\Delta x$ is the spatial resolution of the problem, then the wavefunction at the 


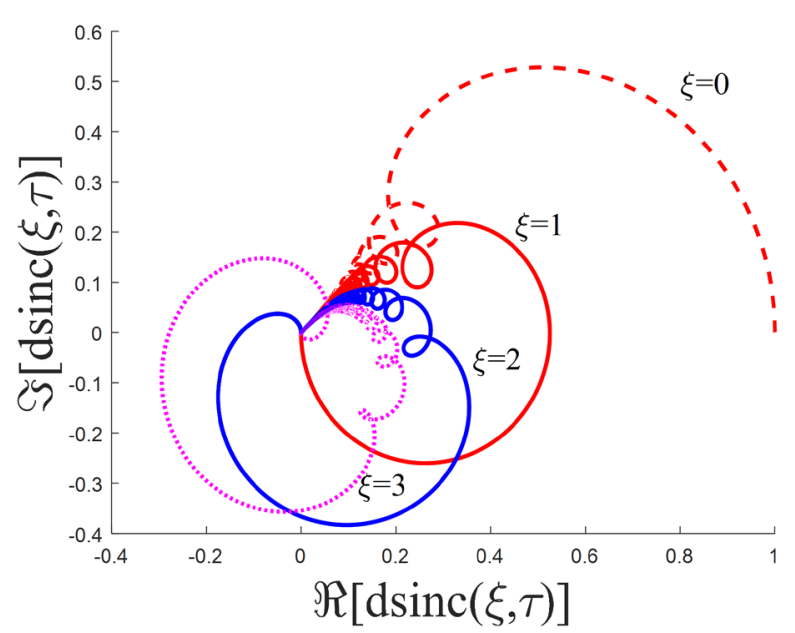

Figure 2. The relation between the real and imaginary parts of the dsinc function for the discrete values $\xi=0,1,2,3$.

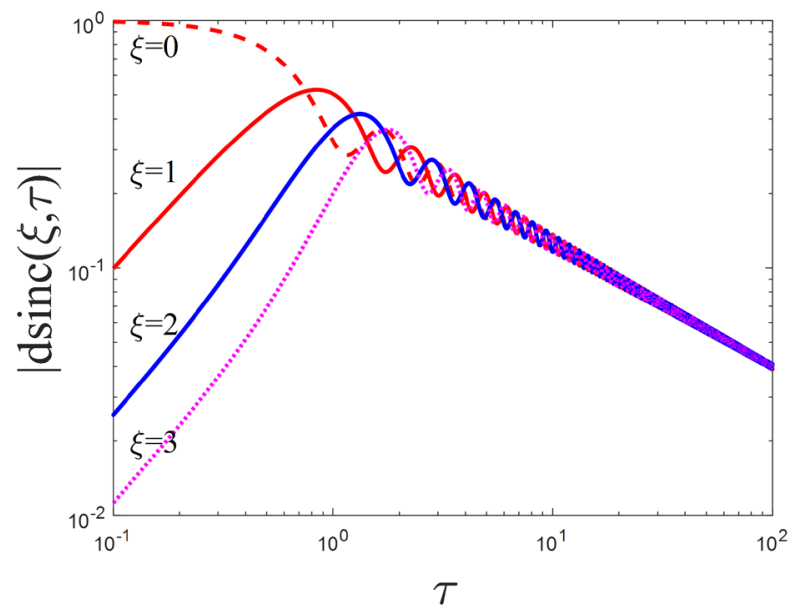

Figure 3. The temporal dependence of the absolute value of dsinc for the discrete values $\xi=0,1,2,3$.

center of the $m$ th point, i.e., at $\xi=m$, is a simple discrete convolution

$$
\psi(m, \tau)=\sum_{n=-\infty}^{\infty} \psi_{n} h(m-n)=\psi_{m}+\sum_{n=-\infty}^{\infty} \psi_{n} \delta h(m-n)
$$

where

$$
h(n) \equiv \mathrm{d} \operatorname{sinc}(n, \tau) \text { and } \delta h(n) \equiv \mathrm{d} \operatorname{sinc}(n, \tau)-\delta(n) .
$$

Moreover, since

$$
\left.\frac{\partial^{2} \operatorname{sinc}(\xi)}{\partial \xi^{2}}\right|_{\tau=n \neq 0}=\frac{2}{n^{2}}(-1)^{n+1} \text { and }\left.\frac{\partial^{2} \operatorname{sinc}(\xi)}{\partial \xi^{2}}\right|_{\tau=0}=-\frac{\pi^{2}}{3},
$$

then Equation (10) can be written as a linear set of differential Equations

$$
\frac{\mathrm{d} \psi(m, \tau)}{\mathrm{d} \tau}=i \sum_{n} w(m-n) \psi(n, \tau) \equiv i w(m) * \psi(m, \tau)
$$


with the universal (and dimensionless) vector

$$
w(m) \equiv\left\{\begin{array}{ll}
(-1)^{m+1} / m^{2} & m \neq 0 \\
-\pi^{2} / 6 & m=0
\end{array},\right.
$$

and the asterisk stands for discrete convolution.

Note that $\sum_{m=-\infty}^{\infty} w(m)=0$ due to Euler's formula [19] [20].

This Equation is universal in the sense that the vector $w(m)$ is time independent. This is a unique property of the sinc pulses, which does not exist in other sets of orthogonal pulses (like rectangular pulses).

Moreover, since the Schrödinger dynamics is a unitary operation, normalisation is kept and there is no change in the wavefunction spectrum. Therefore, Equation (15) is valid for any given time.

In a matrix form, Equation (15) can be written

$$
\frac{\mathrm{d}}{\mathrm{d} \tau}\left(\begin{array}{c}
\vdots \\
\psi(-2, \tau) \\
\psi(-1, \tau) \\
\psi(0, \tau) \\
\psi(1, \tau) \\
\psi(2, \tau) \\
\vdots
\end{array}\right)=i\left(\begin{array}{ccccccc}
\ddots & & & \vdots & & & \\
& -\pi^{2} / 6 & 1 & -2^{-2} & 3^{-2} & & \\
& 1 & -\pi^{2} / 6 & 1 & -2^{-2} & 3^{-2} & \\
\ldots & -2^{-2} & 1 & -\pi^{2} / 6 & 1 & -2^{-2} & \ldots \\
& 3^{-2} & -2^{-2} & 1 & -\pi^{2} / 6 & 1 & \\
& & 3^{-2} & -2^{-2} & 1 & -\pi^{2} / 6 & \\
. & & & \vdots & & & \ddots
\end{array}\right)\left(\begin{array}{c}
\vdots \\
\psi(-2, \tau) \\
\psi(-1, \tau) \\
\psi(0, \tau) \\
\psi(1, \tau) \\
\psi(2, \tau) \\
\vdots
\end{array}\right)
$$

i.e.,

$$
\frac{\mathrm{d}}{\mathrm{d} \tau} \psi=i M \psi
$$

where $M$ is a matrix with the coefficients

$$
M(m, n)= \begin{cases}-\pi^{2} / 6 & n=m \\ (-1)^{n-m+1} /(n-m)^{2} & n \neq m\end{cases}
$$

The nonlocality of this form is clearly emphasized, when compared to the ordinary numerical form of the Schrödinger Equation with the ordinary 1D Cartesian local Laplacian

$$
\frac{\mathrm{d}}{\mathrm{d} \tau}\left(\begin{array}{c}
\vdots \\
\psi(-2, \tau) \\
\psi(-1, \tau) \\
\psi(0, \tau) \\
\psi(1, \tau) \\
\psi(2, \tau) \\
\vdots
\end{array}\right)=i\left(\begin{array}{ccccccc}
\ddots & & & \vdots & & & \cdot \\
& -2 & 1 & & & & \\
& 1 & -2 & 1 & & & \\
\cdots & & 1 & -2 & 1 & & \cdots \\
& & & 1 & -2 & 1 & \\
& & & & 1 & -2 & \\
. & & & \vdots & & & \ddots
\end{array}\right)\left(\begin{array}{c}
\vdots \\
\psi(-2, \tau) \\
\psi(-1, \tau) \\
\psi(0, \tau) \\
\psi(1, \tau) \\
\psi(2, \tau) \\
\vdots
\end{array}\right)
$$

In the presence of a non-zero potential, whose maximum spatial frequencies is also lower than $1 / 2 \Delta x$ the Schrödinger Equation can be rewritten in a matrix form

$$
\frac{\mathrm{d}}{\mathrm{d} \tau} \boldsymbol{\psi}=i(M-V) \psi
$$

where 


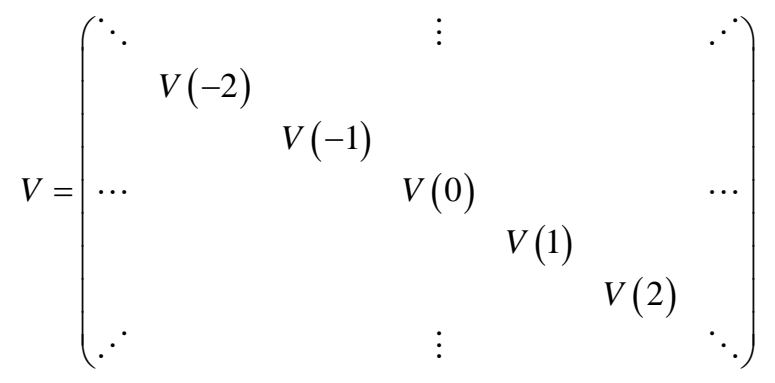

or simply

$$
V(n, m)=V(n) \delta(n-m) .
$$

Therefore, a pulse which is initially located at $x=0$ has an instantaneous effect over the entire space, and its effect on any other point (say $n \Delta x$ ) is inversely proportional to the distance between them, i.e., $(n \Delta x)^{-2}$.

On the other hand, when the local Laplacian is used, i.e., (20), then the pulse effect on a point $n \Delta x$ afar, would be felt only after $n$ consecutive steps. Therefore, if there is a barrier (i.e., non zero potential) between these points $(x=0$ and $x=n \Delta x)$ then with a local Laplacian it may seem that the effect of the one on the other (and vice versa) must take into account the barrier in between the two points. However, in fact, as the nonlocal form teaches, in the short time its effect is negligible since the Schrödinger Equation can be approximated by

$$
\frac{\mathrm{d}}{\mathrm{d} \tau}\left(\begin{array}{c}
\psi(n, \tau) \\
\psi(m, \tau)
\end{array}\right)=-i\left(\begin{array}{cc}
\pi^{2} / 6+V(n) & (-1)^{n-m} /(n-m)^{2} \\
(-1)^{n-m} /(n-m)^{2} & \pi^{2} / 6+V(m)
\end{array}\right)\left(\begin{array}{c}
\psi(n, \tau) \\
\psi(m, \tau)
\end{array}\right),
$$

and its short-time solution

$$
\left(\begin{array}{l}
\psi(n, \tau) \\
\psi(m, \tau)
\end{array}\right)=\left(\begin{array}{cc}
1-i \tau\left[\pi^{2} / 6+V(n)\right] & -i \tau(-1)^{n-m} /(n-m)^{2} \\
-i \tau(-1)^{n-m} /(n-m)^{2} & 1-i \tau\left[\pi^{2} / 6+V(m)\right]
\end{array}\right)\left(\begin{array}{l}
\psi(n, 0) \\
\psi(m, 0)
\end{array}\right)
$$

shows that in the short time the potential has only a local effect (provided it is a smooth function), i.e., $\psi(n, \tau)$ is affected only by $V(n)$ (and the effect is a simple phase change). However, the wavefunction has a nonlocal effect, i.e., $\psi(n, \tau)$ is affected by any non zero $\psi(m, \tau)$ (for any $m$ ). This result is consistent with Ref. [21], where it was demonstrated that in short time, singular wavefunction are unaffected by the barrier despite their nonlocal effect.

\section{Inverse Square Law}

By multiplying Equation (15) with the complex conjugate of the wavefunction and taking the real part of the Equation one finds a nonlocal Equation for the probability density

$$
\frac{\mathrm{d}|\psi(m, \tau)|^{2}}{\mathrm{~d} \tau}=-2 \sum_{n \neq m} \frac{(-1)^{m-n+1}}{(m-n)^{2}} \mathfrak{J}\left\{\psi(n, \tau) \psi^{*}(m, \tau)\right\}
$$

Using the notation $\psi(n, \tau) \equiv A_{n} \exp \left(i \phi_{n}\right)$ then Equation (26) is simply

$$
\frac{\mathrm{d} A_{m}^{2}}{\mathrm{~d} \tau}=2 \sum_{n \neq m} \frac{(-1)^{m-n} A_{n} A_{m} \sin \left(\phi_{n}-\phi_{m}\right)}{(m-n)^{2}}
$$


and the equivalent phase Equation reads

$$
\frac{\mathrm{d} \phi_{m}}{\mathrm{~d} \tau}=-\sum_{n \neq m} \frac{(-1)^{m-n}}{(m-n)^{2}} \frac{A_{n}}{A_{m}} \cos \left(\phi_{n}-\phi_{m}\right)-\frac{\pi^{2}}{6}
$$

If $\psi(n, \tau)$ is presented as a $2 \mathrm{D}$ vector in a $3 \mathrm{D}$ space

$$
\psi(n, \tau) \equiv \widehat{x} \Re \psi(n, \tau)+\widehat{y} \mathfrak{\Im} \psi(n, \tau)
$$

instead of a complex number in a complex plane, then the numerator in the summation can be presented as the cross product of two vectors, i.e.,

$$
\frac{\mathrm{d}\|\boldsymbol{\psi}(m, \tau)\|^{2}}{\mathrm{~d} \tau}=2 \sum_{n \neq m} \frac{(-1)^{m-n}[\psi(n, \tau) \times \psi(m, \tau)] \cdot \hat{z}}{(m-n)^{2}}
$$

In this terminology, $\|\psi(m, \tau)\|$ is the norm of the vector $\psi(m, \tau)$ and the cross represents cross (3D vectors) product.

It is instructive to see the resemblance between this law and any other inverse square law.

Equation (30) can also be written in terms of the derivative of the vector's norm $\|\psi(m, \tau)\|$

$$
\frac{\mathrm{d}\|\psi(m, \tau)\|}{\mathrm{d} \tau}=\sum_{n \neq m} \frac{(-1)^{m-n}\left[\psi(n, \tau) \times \hat{\boldsymbol{\psi}}_{m}\right] \cdot \hat{z}}{(m-n)^{2}}
$$

where

$$
\hat{\psi}_{m} \equiv \psi(m, \tau) /\|\psi(m, \tau)\|
$$

is the unit vector in the $\psi(m, \tau)$ direction.

It is therefore clear, that maximum probability density transfer occurs when the relative phase between the two points is $\pi / 2$, i.e. when the "vectors" $\psi(n, \tau)$ and $\psi(m, \tau)$ are orthogonal.

In Figure 4 such a density transfer is illustrated. In this case the wavefunction

$\psi(\xi, \tau=0)=N[\operatorname{sinc}(\xi-8)+i \operatorname{sinc}(\xi-9)]$ was taken as the initial state $(N$ is the normalisation constant). As can be seen, in the short time regime ( $\tau=0.198$ in this case), the probability was transferred from the pulse at $\xi=9$ to the one at $\xi=8$.

Moreover, it is clear from Equation (27) and Equation (30) that maximum probability transfer to a certain location (say $\xi=0$ ) occurs (provided the initial state is bounded) when the initial state oscillates in signs, i.e.,

$$
\psi(\xi, \tau=0)=i \sum_{n \neq 0}(-1)^{n} \operatorname{sinc}(\xi-n)+\operatorname{sinc}(\xi)
$$

In this case the rate in which the probability increases (or decreases in the opposite case) is exactly $2 \pi^{2} / 3$ since (using Euler formula, see Ref. [19] [20])

$$
\frac{\mathrm{d} \ln \left[|\psi(m, \tau)|^{2}\right]}{\mathrm{d} \tau}=2 \sum_{n \neq m} \frac{1}{(m-n)^{2}}=2 \frac{\pi^{2}}{3}
$$

and the probability density at $\xi=0$ can increase almost four fold before it start to decay (see Figure 5). 

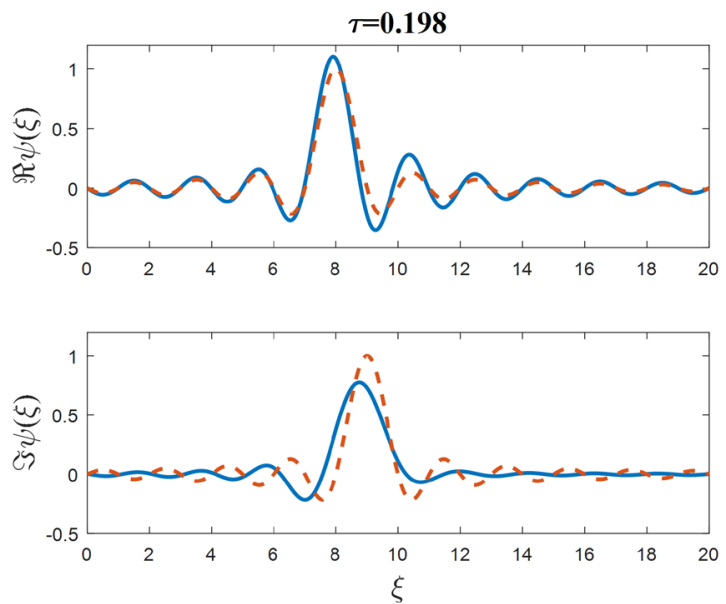

Figure 4. Illustration of probability transfer. The dashed curve represents the initial state $\psi(\xi, \tau=0)=\operatorname{sinc}(\xi-8)+i \operatorname{sinc}(\xi-9)$, while the solid curve stands for $\psi(\xi, \tau=0.198)=\mathrm{d} \operatorname{sinc}(\xi-8, \tau)+i \mathrm{~d} \operatorname{sinc}(\xi-9, \tau)$. In both cases the real part is plotted in the upper panel, while the imaginary part is plotted in the lower one.
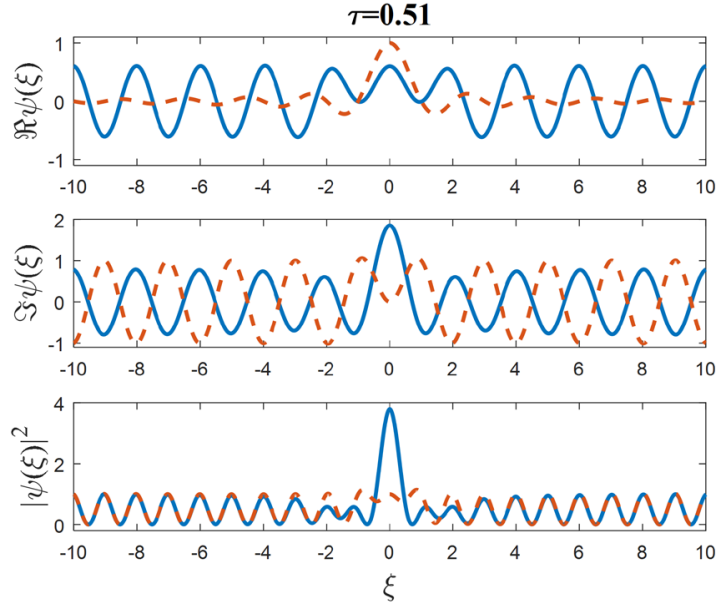

Figure 5. The short time dynamics of the wavefunction (32). The dashed curves represent the initial ( $\tau=0$ ) state, while the solid curves represent the state after a period of $\tau=0.51$, where the probability density at $\xi=0$ increases by a factor of 4 .

The source of this nonlocality is the fact that each one of the sinc is spread over the entire space. However, the important result is, that this nonlocal presentation of the Schrödinger Equation is universal and it is independent of $\Delta x$, which can be as short as the spatial measurement's accuracy.

\section{Conclusions}

It has been shown that when a given wavefunction is spectrally bounded, then the Schrödinger dynamics can be formulated in a universal nonlocal form. Instead of a local partial differential Equation, it can be formulated as an infinite set of ordinary differential Equation, where the coupling is pure numbers, which 
are strongly related to Euler's formula $\sum_{n=1}^{\infty} n^{2}=\pi^{2} / 6$.

Therefore, the mutual effect of every two points on the wavefunction is instantaneous and can be formulated by an inverse square law.

\section{Conflicts of Interest}

The author declares no conflicts of interest regarding the publication of this paper.

\section{References}

[1] Crank, J. (1975) The Mathematics of Diffusion. Clarendon Press, Oxford.

[2] Garca-Calderon, G., Rubio, A. and Villavicencio, J. (1999) Low-Energy Relativistic Effects and Nonlocality in Time-Dependent Tunneling. Physical Review A, 59, 1758. https://doi.org/10.1103/PhysRevA.59.1758

[3] Delgado, F., Muga, J.G., Ruschhaupt, A., Garcia-Calderon, G. and Villavicencio, J. (2003) Tunneling Dynamics in Relativistic and Nonrelativistic Wave Equations. Physical Review A, 68, Article ID: 032101. https://doi.org/10.1103/PhysRevA.68.032101

[4] Granot, E. and Marchewka, A. (2011) Emergence of Currents as a Transient Quantum Effect in Nonequilibrium Systems. Physical Review A, 84, 032110-032115. https://doi.org/10.1103/PhysRevA.84.032110

[5] Granot, E. and Marchewka, A. (2005) Generic Short-Time Propagation of SharpBoundaries Wave Packets. Europhysics Letters, 72, 341-347. https://doi.org/10.1209/epl/i2005-10264-2

[6] Schweizer, W. (2002) Numerical Quantum Dynamics. In: Progress in Theoretical Chemistry and Physics, Volume 9, Kluwer Academic Publishers, Dordrecht. https://doi.org/10.1007/0-306-47617-7

[7] Marchildon, L. (2002) Quamtum Mechanics-From Basics Principles to Numerical Methods and Applications. Springer-Verlag, Berlin, Heidelberg.

[8] Feynman, R.P. and Hibbs, A.R. (1965) Quantum Mechanics and Path Integrals. McGraw-Hill Companies, London.

[9] Kleber, M. (1994) Exact Solutions for Time-Dependent Phenomena in Quantum Mechanics. Physics Reports, 236, 331-393. https://doi.org/10.1016/0370-1573(94)90029-9

[10] Bender, C.M. and Orszag, S.A. (1978) Advanced Mathematical Methods for Scientists and Engineers. McGraw Hill, Singapore.

[11] Oppenheim, A.V. and Schafer, R.W. (1975) Digital Signal Processing. Pearson, London.

[12] Soto, M.A., Alem, M., Shoaie, M.A., Vedadi, A., Brès, C.-S., Thévenaz, L. and Schneider, T. (2013) Optical Sinc-Shaped Nyquist Pulses of Exceptional Quality. Nature Communications, 4, 2898. https://doi.org/10.1038/ncomms3898

[13] Schmogrow, R., Bouziane, R., Meyer, M., Milder, P.A., Schindler, P.C., Killey, R.I., Bayvel, P., Koos, C., Freude, W. and Leuthold, J. (2012) Real-Time OFDM or Nyquist Pulse Generation-Which Performs Better with Limited Resources? Optics Express, 20, B543. https://doi.org/10.1364/OE.20.00B543

[14] Hirooka, T., Ruan, P., Guan, P. and Nakazawa, M. (2012) Highly Dispersion-Tolerant 160 Gbaud Optical Nyquist pulse TDM Transmission over 525km. Optics EX press, 20, 15001-15007. https://doi.org/10.1364/OE.20.015001 
[15] Hirooka, T. and Nakazawa, M. (2012) Linear and Nonlinear Propagation of Optical Nyquist Pulses in Fibers. Optics Express, 20, 19836-19849. https://doi.org/10.1364/OE.20.019836

[16] Schmogrow, R., et al. (2012) 512QAM Nyquist Sinc-Pulse Transmission at 54 Gbit/s in an Optical Bandwidth of $3 \mathrm{GHz}$. Optics Express, 20, 6439-6447. https://doi.org/10.1364/OE.20.006439

[17] Bosco, G., Carena, A., Curri, V., Poggiolini, P. and Forghieri, F. (2010) Performance Limits of Nyquist-WDM and CO-OFDM in High-Speed PM-QPSK Systems. IEEE Photonics Technology Letters, 22, 1129-1131. https://doi.org/10.1109/LPT.2010.2050581

[18] Granot, E. (2018) Information Loss in Quantum Dynamics, Advanced Technologies of Quantum Key Distribution Sergiy Gnatyuk. IntechOpen, London.

https://doi.org/10.5772/intechopen.70395 https://www.intechopen.com/books/advanced-technologies-of-quantum-key-distrib ution/information-loss-in-quantum-dynamics

[19] Ayoub, R. (1974) Euler and the Zeta Function. The American Mathematical Monthly, 81, 1067-1086. https://doi.org/10.1080/00029890.1974.11993738

[20] Granot, E. (2019) Derivation of Euler's Formula and $\zeta(2 \mathrm{k})$ Using the NyquistShannon Sampling Theorem. American Journal of Signal Processing, 9, 1-5. http://article.sapub.org/10.5923.j.ajsp.20190901.01.html

[21] Granot, E. and Marchewka, A. (2007) Universal Potential-Barrier Penetration by Initially Confined Wave Packets. Physical Review A, 76, Article ID: 012708. https://doi.org/10.1103/PhysRevA.76.012708 